\title{
Understanding diagnostic tests. Part 1.
} \section{$B P$}

\section{PRACTICAL SCENARIO}

Investigators studied the diagnostic accuracy of serum procalcitonin levels to diagnose parapneumonic pleural effusions (PPE) and differentiate it from other causes of pleural effusions. They found that procalcitonin (with a cut-off value of $0.195 \mathrm{ng} / \mathrm{mL}$ ) had a sensitivity of $83 \%$ and a specificity of $80 \%$ to diagnose PPE and accurately diagnosed individuals with PPE. ${ }^{(1)}$

\section{USING DIAGNOSTIC TESTS IN CLINICAL PRACTICE}

Clinicians are frequently faced with the challenge of diagnosing a disease based on diagnostic test results. Most diagnostic tests used in clinical practice, however, are not perfect and produce false positive results (the test is positive, but the patient does not have the disease) and false negative results (the test is negative, but the patient has the disease). Therefore, learning to interpret the properties of diagnostic tests is a critical competency for clinicians and researchers. In this article, we discuss sensitivity and specificity. In the forthcoming parts, we will discuss positive and negative predictive values, and receiver operating characteristic (ROC) curves.

Sensitivity and specificity are important measures of a diagnostic test because they give us an idea of how well a new diagnostic test performs when compared with an existing gold standard test. Sensitivity is defined as the proportion of subjects with the disease who have a positive test. In the example in Table 1 , true positives ( $n=39$ ) divided by the total number of subjects with disease $(n=47)$ results in $83 \%$. Specificity is defined as the proportion of subjects without the disease who have a negative test. In the example, true negatives ( $n$ $=81$ ) divided by total number of subjects without the disease $(n=101)$ results in $80 \%$.
When a new diagnostic test is evaluated, the investigator sets a cut-off point which defines whether the test is positive or negative, and there is always a trade-off between sensitivity and specificity. In our example, if the cut-off point for a positive procalcitonin test was decreased from $0.195 \mathrm{ng} / \mathrm{mL}$ to $0.095 \mathrm{ng} / \mathrm{mL}$, it might detect more cases of PPE, decreasing the false negative rate and increasing sensitivity, but the test would also be positive in more subjects without PPE, increasing the false positive rate and decreasing specificity. This trade-off between sensitivity and specificity for several possible cut-off points can be used to plot a ROC curve and describe the overall test performance in discriminating between presence and absence of the disease; we can also use sensitivity and specificity to calculate likelihood ratios, as we will see later in this series.

Sensitivity and specificity are useful measures to evaluate the performance of a diagnostic test but are not very helpful for personalized clinical decision making. ${ }^{(2)}$ When a clinician is facing a patient with a positive test result, the most important question is: what is the probability that, given that the test is positive, the patient has the disease? The sensitivity of the test does not tell us that; it tells us the probability of a positive test, given that the patient has the disease. We will address more relevant clinical measures of diagnosis in part 2 of this series.

Table 1. Diagnostic performance of serum procalcitonin testing for identifying parapneumonic pleural effusion.

\begin{tabular}{cccc} 
Result & \multicolumn{2}{c}{ PPE } & Total \\
& + & - & \\
PCT+ & $\mathrm{a}=39$ & $\mathrm{~b}=20$ & 59 \\
PCT- & $\mathrm{c}=8$ & $\mathrm{~d}=81$ & 89 \\
Total & 47 & 101 & 148 \\
\hline
\end{tabular}

Data obtained from He et al.(1) PCT: procalcitonin; PPE: parapneumonic pleural effusion. Sensitivity (light grey column $)=a /(a+c)$. Specificity (dark grey column $)=$ $b /(b+d)$.

\section{REFERENCES}

1. He C, Wang B, Li D, Xu H, Shen Y. Performance of procalcitonin in diagnosing parapneumonic pleural effusions: A clinical study and meta-analysis. Medicine (Baltimore). 2017;96(33):e7829. https://do

org/10.1097/MD.0000000000007829

2. Altman DG, Bland M. Diagnostic tests. 1: Sensitivity and specificity. BMJ. 1994;308(6943):1552. https://doi.org/10.1136/bmj.308.6943.1552

1. Methods in Epidemiologic, Clinical, and Operations Research-MECOR-program, American Thoracic Society/Asociación Latinoamericana del Tórax Montevideo, Uruguay.

2. Divisão de Pneumologia, Instituto do Coração, Hospital das Clínicas Faculdade de Medicina, Universidade de São Paulo, São Paulo (SP) Brasil.

3. Department of Preventive Medicine, Keck School of Medicine, University of Southern California, Los Angeles, CA, USA 


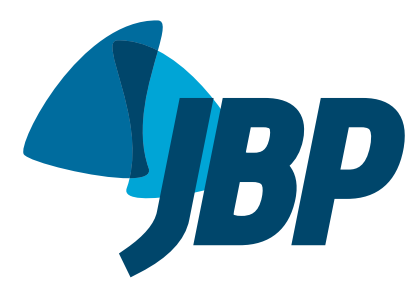

Manuscript: Understanding diagnostic tests. Part 1.

Publication: J Bras Pneumol. 2017;43(5):330

DOI: http://dx.doi.org/10.1590/S1806-37562017000000330

On page 330 of the original publication, in Table 1, last line of the text, where is written Specificity (dark grey column) $=b /(b+d)$

Should be read

Specificity (dark grey column) $=d /(b+d)$ 